\title{
On the numerical solution of generalized nonlinear Schrodinger equation using RBFS
}

Marjan Uddin and Sirajul Haq 


\title{
ON THE NUMERICAL SOLUTION OF GENERALIZED NONLINEAR SCHRODINGER EQUATION USING RBFS
}

\author{
MARJAN UDDIN AND SIRAJUL HAQ
}

Received 21 February, 2012

\begin{abstract}
A meshfree technique based on radial basis functions (RBFs) is applied for the numerical solution of generalized nonlinear Schrodinger equation. The spatial derivatives are approximated by using the derivative of interpolation and a low order forward scheme is used to approximate the temporal derivative. Three test problems concerning the motion of solitary wave, interaction of two solitary waves and solution that blows up in finite time respectively are investigated, which examined the accuracy of the method in terms of the $\mathrm{L}_{2}, \mathrm{~L}_{\infty}$ error norms, and the conservative quantities, $I_{1}, I_{2}$ and $I_{3}$. The results obtained from the method are compared with the exact solution and the earlier work in the literature.
\end{abstract}

2010 Mathematics Subject Classification: 65M06; 65M20

Keywords: generalized nonlinear Schrodinger equation, meshfree collocation method, radial basis functions

\section{INTRODUCTION}

The generalized nonlinear Schrodinger (GNS) equation given by

$i \frac{\partial w}{\partial t}+\frac{\partial^{2} w}{\partial x^{2}}+q_{1}|w|^{2} w+q_{2}|w|^{4} w+i q_{3} \frac{\partial\left(|w|^{2}\right)}{\partial x} w+i q_{4}|w|^{2} \frac{\partial w}{\partial x}=0,-\infty<x<\infty$,

where $i=\sqrt{-1}, w$ is a complex valued function of the spatial coordinate $x$ and time $t$, the parameters $q_{1}, q_{2}, q_{3}$ and $q_{4}$ are real constants. The general solution of Eq. (1.1) does not belong to the class of integrable equations. However some cases of Eq. (1.1) are completely integrable, and has application in fluid mechanics, nonlinear optics and plasma physics (see [21,22] and references therein). For the coefficients $q_{1} \neq 0, q_{2} \neq 0$ and $q_{3}=q_{4}=0$ Eq. (1.1) reduces to nonlinear Schrodinger (NS) Equation. For certain values of the coefficients and some initial condition the solution of GNS equation experience finite time blow up [21]. Under the assumption that the solution $|w| \rightarrow 0$ as $|x| \rightarrow \infty$ GNS equation possess infinite number of conservation laws. However, rapidly decaying solution to Eq. (1.1) have three conservative laws 
[21,22] given by

$$
\begin{aligned}
I_{1} & =\int_{-\infty}^{\infty}|w|^{2} d x \\
I_{2} & =\int_{-\infty}^{\infty}\left[\left|w_{x}\right|^{2}-\frac{1}{2}\left(2 q_{3}+q_{4}\right)|w|^{2} \operatorname{Im}\left(w w_{x}^{*}\right)\right. \\
& \left.-\frac{1}{2} q_{1}|w|^{4}+\frac{1}{6}\left[q_{3}\left(2 q_{3}+q_{4}\right)-2 q_{2}\right]|w|^{6}\right] d x, \\
I_{3} & =\int_{-\infty}^{\infty}\left[2 \operatorname{Im}\left(w w_{x}^{*}\right)-q_{3}|w|^{4}\right] d x .
\end{aligned}
$$

Although the GNS equation has been studied by many researchers (see [1, 12] and references therein), only a few numerical methods have been applied to solve the GNS equation. D. Pathria and J. LI. Morris [22] used the pseudo-spectral methods to obtain the solution of GNS equation, and discussed some of its properties, M. P. Robinson [23] used orthogonal spline collocation method for numerical solution of GNS equation. In some numerical studies of GNS equation the contribution from the nonlinear derivatives have been ignored [3, 4, 24]. More recently G. M. Muslu and H. A. Erbay [19] have been used Higher-order split-step method for the solution of GNS equation.

In the last decade, the theory of radial basis functions (RBFs) has enjoyed a great success as scattered data interpolating technique. A radial basis function, $\phi\left(x-x_{j}\right)=$ $\phi\left(\left\|x-x_{j}\right\|\right)$, is a continuous spline which depends upon the separation distances of a subset of data centers, $X \subset \mathfrak{R}^{n},\left\{x_{j} \in X, j=1,2, \ldots, N\right\}$. Due to spherical symmetry about the centers $x_{j}$, the RBFs are called radial. The distances, $\left\|x-x_{j}\right\|$, are usually taken to be the Euclidean metric. Hardy [11] was the first to introduced a general scattered data interpolation method, called radial basis functions method for the approximation of two-dimensional geographical surfaces. In 1982 Franke [8] in a review paper made the comparison among all the interpolation methods for scattered data sets available at that time, and the radial basis functions outperformed all the other methods regarding efficiency, stability and ease of implementations. Franke found that Hardy's multiquadrics (MQ) were ranked the best in accuracy, followed by thin plate splines (TPS). Despite MQ's excellent performance, it contains a shape parameter $c$, and the accuracy of MQ is greatly affected by the choice of shape parameter $c$ whose optimal value is still unknown. Franke[7] used the formula $c^{2}=(1.25)^{2} d^{2}$ where $d$ is the mean distance from each data point to its nearest neighbor. Hickernell and Hon [13] and Golberg et al. [9] had successfully used the technique of crossvalidation to obtain an optimal value of the shape parameter. In 1990 radial basis functions scheme was introduced by Kansa [14] to solve partial differential equations. The existence, uniqueness and convergence of this method was discussed by Micchelli [18], Madych [17], Frank and Schaback [6]. It was studied by Micchelli in 
1986 that for distinct interpolation points system obtained in multiquadric (MQ) method is always solvable. Very recently RBFs method have been used for the solutions of nonlinear PDEs (see [10,25,26]). Nicolas ali libre et.al [16] have used an adaptive scheme for nearly singular PDEs, Emdadi et. al [5] proposed a stable PDE solution method for large multiquadric shape parameters whereas a modified meshless control volume method was proposed by P. Orsini, H. Power [20]. G. Kosec and B. Sarler [15] have used local RBF collocation method for Darcy flow. S.N. Atluri et.al [2] have developed a meshless finite volume method through MLPG mixed approach. The RBFs scheme is truly a meshfree method which does not require the generation of a mesh, and since the MQ is infinitely differentiable, we can approximate the higher order spatial derivative directly by computing the derivative of the basis functions. However the RBFs method faces a serious ill-conditioning problem when we use RBFs as a global interplant and increases the number of collocation points. There are different ways to overcome this problem e.g to use domain decomposition technique.

In this work, we apply meshfree collocation method based on three radial basis functions, MQ $\sqrt{r^{2}+c^{2}}$ where $c$ is a shape parameter, Spline basis $\left(\mathrm{r}^{5}\right)$ and TPS $\left(r^{4} \log (r)\right)$ for the numerical solution of generalized nonlinear Schrodinger (GNS) equation. GNS equation has an exact traveling solitary wave solution [19]

$$
\begin{gathered}
w(x, t)=\left[\frac{4}{4+3 \sinh ^{2}(x-2 t-15)}\right]^{1 / 2} \exp [i \phi(x, t)], \\
\phi(x, t)=2 \tanh ^{-1}\left[\frac{1}{2} \tanh (x-2 t-15)\right]+x-15 .
\end{gathered}
$$

This solitary wave is centered at $x_{0}=15$ and moving to the right with the velocity 2 .

To avoid complex computation, we transform the GNS equation (1.1) into a nonlinear coupled equations, by decomposing $w$ into its real and imaginary parts,

$$
w(x, t)=u(x, t)+i v(x, t), i^{2}=-1,
$$

where $u(x, t)$ and $v(x, t)$ are real functions. As a result we obtained the following coupled pair of equations

$$
\begin{aligned}
& \frac{\partial u}{\partial t}+\frac{\partial^{2} v}{\partial x^{2}}+q_{1}\left(u^{2}+v^{2}\right) v+q_{2}\left(u^{2}+v^{2}\right)^{2} v \\
& +q_{3}\left(\frac{\partial\left(u^{2}+v^{2}\right)}{\partial x}\right) u+q_{4}\left(u^{2}+v^{2}\right) \frac{\partial u}{\partial x}=0 \\
& -\frac{\partial v}{\partial t}+\frac{\partial^{2} u}{\partial x^{2}}+q_{1}\left(u^{2}+v^{2}\right) u+q_{2}\left(u^{2}+v^{2}\right)^{2} u \\
& -q_{3}\left(\frac{\partial\left(u^{2}+v^{2}\right)}{\partial x}\right) v-q_{4}\left(u^{2}+v^{2}\right) \frac{\partial v}{\partial x}=0 .
\end{aligned}
$$


The structure of the present paper is organized as follows. In Section 2 we discuss the meshfree method. In Section 3 we deal the stability analysis. Section 4 is devoted to the numerical tests of the method on the problems related to the GNS equation. In Section 5, the results are concluded.

\section{ANALYSIS OF THE METHOD}

In this section, we consider a general time dependent boundary value problem

$$
\frac{\partial u}{\partial t}+\mathscr{L} u=f(x, t), x \in \Omega, \quad \mathscr{B} u=g(x), \quad x \in \partial \Omega,
$$

where $\mathscr{L}$ and $\mathscr{B}$ are derivative and boundary operators respectively. $\Omega$ and $\partial \Omega$ represent interior and boundary of the domain respectively. Eq. (2.1) has to be supplemented by initial condition of the form $u(x, 0)=u_{0}(x)$. We use $\theta$-weighted scheme for temporal derivative in the following form

$$
\frac{u^{(n+1)}-u^{(n)}}{\delta t}+\theta \mathscr{L} u^{(n+1)}+(1-\theta) \mathscr{L} u^{(n)}=f\left(x, t^{(n+1)}\right) .
$$

In the above equation $\delta t$ is the time step, $u^{(n)}$ ( $n$ is non-negative integer) is the solution at time $t^{(n)}=n \delta t, 0 \leq \theta \leq 1$. Let $\left\{x_{i}\right\}_{i=1}^{N_{d}}$ and $\left\{x_{i}\right\}_{i=N_{d}+1}^{N}$ be respectively interior and boundary points among the collocation points $\left\{x_{i}\right\}_{i=1}^{N}$ in the domain. The solution of Eq. (2.1) can be approximated by

$$
u^{(n)}\left(x_{i}\right)=\sum_{j=1}^{N} \psi\left(r_{i j}\right) \lambda_{j}^{(n)} .
$$

In the above eqution $\psi\left(r_{i j}\right)$ are radial basis functions with Euclidean norm $r_{i j}=$ $\left\|P_{i}-P_{j}\right\|$ between the points $P_{i}$ and $P_{j},\left\{\lambda_{j}\right\}_{j=1}^{N}$ are constants to be determined. From Eqs. (2.2) and (2.3), we can write

$$
\begin{gathered}
\sum_{j=1}^{N}\left(\frac{\psi\left(r_{i j}\right) \lambda_{j}^{(n+1)}-\psi\left(r_{i j}\right) \lambda_{j}^{(n)}}{\delta t}+\theta \mathscr{L} \psi\left(r_{i j}\right) \lambda_{j}^{(n+1)}+(1-\theta) \mathscr{L} \psi\left(r_{i j}\right) \lambda_{j}^{(n)}\right) \\
=f\left(x, t^{(n+1)}\right), i=1,2, \ldots, N_{d}, \\
\sum_{j=1}^{N} \mathcal{B} \psi\left(r_{i j}\right) \lambda_{j}^{(n+1)}=g\left(x_{i}, t^{(n+1)}\right), i=N_{d}+1, \ldots, N,
\end{gathered}
$$

where $r_{i j}=\left\|P_{i}-P_{j}\right\|$. Eqs (2.4)-(2.5) are $N$ equations in $N$ unkhown $\left\{\lambda_{j}\right\}_{j=1}^{N}$, which can be solved by using Gauss elimination method. We use $\theta=0.5$ in our work. 


\subsection{GNS equation}

The decomposed form of GNS equation can be written as

$$
\begin{gathered}
u_{t}+\left(2 q_{3}+q_{4}\right) u^{2} u_{x}=-v_{x x}-q_{1}\left(u^{2}+v^{2}\right) v \\
-q_{2}\left(u^{2}+v^{2}\right)^{2} v-2 q_{3} u v v_{x}-q_{4} v^{2} u_{x}, \\
v_{t}+\left(2 q_{3}+q_{4}\right) v^{2} v_{x}=u_{x x}+q_{1}\left(u^{2}+v^{2}\right) u \\
+q_{2}\left(u^{2}+v^{2}\right)^{2} u-2 q_{3} u v u_{x}-q_{4} u^{2} v_{x}, a \leq x \leq b,
\end{gathered}
$$

where $q_{1}, q_{2}, q_{3}$ and $q_{4}$ are real constants.

The boundary conditions are

$$
\begin{aligned}
& u(a, t)=f_{1}(a, t), u(b, t)=f_{2}(b, t), \\
& v(a, t)=g_{1}(a, t), v(b, t)=g_{2}(b, t), t>0,
\end{aligned}
$$

and initial conditions

$$
u(x, 0)=f(x, 0), \quad v(x, 0)=g(x, 0) \quad a \leq x \leq b .
$$

From Eqs. (2.6), we can write

$$
\begin{gathered}
\left(\frac{u^{(n+1)}-u^{(n)}}{\delta t}\right)+\left(3 q_{3}+q_{4}\right) \theta\left(u^{2} u_{x}\right)^{(n+1)}+\left(3 q_{3}+q_{4}\right)(1-\theta)\left(u^{2} u_{x}\right)^{(n)} \\
=\left(-v_{x x}-q_{1}\left(u^{2}+v^{2}\right) v-q_{2}\left(u^{2}+v^{2}\right)^{2} v-2 q_{3} u v v_{x}-q_{4} v^{2} u_{x}\right)^{(n)}, \\
\left(\frac{v^{(n+1)}-v^{(n)}}{\delta t}\right)+\left(\left(3 q_{3}+q_{4}\right) \theta\left(v^{2} v_{x}\right)^{(n+1)}+\left(3 q_{3}+q_{4}\right)(1-\theta)\left(v^{2} v_{x}\right)^{(n)}\right) \\
=\left(u_{x x}+q_{1}\left(u^{2}+v^{2}\right) u+q_{2}\left(u^{2}+v^{2}\right)^{2} u-2 q_{3} u v u_{x}-q_{4} u^{2} v_{x}\right)^{(n)},
\end{gathered}
$$

where $t^{(n+1)}=t^{(n)}+\delta t$. Rearanging Eqs. (2.10)-(2.11), and substituting the linearised values of the nonlinear terms $\left(u^{2} u_{x}\right)^{(n+1)}$ and $\left(v^{2} v_{x}\right)^{(n+1)}$ given as

$$
\begin{aligned}
& \left(u^{2} u_{x}\right)^{(n+1)}=\left(u^{2}\right)^{(n)} u_{x}^{(n+1)}+2\left(u^{(n)} u_{x}^{(n)}\right) u^{(n+1)}-2\left(u^{2}\right)^{(n)} u_{x}^{(n)}, \\
& \left(v^{2} v_{x}\right)^{(n+1)}=\left(v^{2}\right)^{(n)} v_{x}^{(n+1)}+2\left(v^{(n)} v_{x}^{(n)}\right) v^{(n+1)}-2\left(v^{2}\right)^{(n)} v_{x}^{(n)},
\end{aligned}
$$

we get the following equations

$$
\begin{aligned}
& u^{(n+1)}+\left(3 q_{3}+q_{4}\right) \delta t \theta\left[\left(u^{2}\right)^{(n)} u_{x}^{(n+1)}+2\left(u^{(n)} u_{x}^{(n)}\right) u^{(n+1)}\right] \\
& =u^{(n)}+2\left(3 q_{3}+q_{4}\right) \delta t \theta\left(u^{2} u_{x}\right)^{(n)}-\left(3 q_{3}+q_{4}\right) \delta t(1-\theta)\left(u^{2} u_{x}\right)^{(n)} \\
& +\delta t\left[-\left(v_{x x}\right)^{(n)}-q_{1}\left(\left(u^{2}\right)^{(n)}+\left(v^{2}\right)^{(n)}\right) v^{(n)}-q_{2}\left(\left(u^{2}\right)^{(n)}\right.\right. \\
& \left.\left.+\left(v^{2}\right)^{(n)}\right)^{2} v^{(n)}-2 q_{3}\left(u v v_{x}\right)^{(n)}-q_{4}\left(v^{2} u_{x}\right)^{(n)}\right] \\
& v^{(n+1)}+\left(3 q_{3}+q_{4}\right) \delta t \theta\left[\left(v^{2}\right)^{(n)} v_{x}^{(n+1)}+2\left(v^{(n)} v_{x}^{(n)}\right) v^{(n+1)}\right]
\end{aligned}
$$




$$
\begin{aligned}
& =v^{(n)}+2\left(3 q_{3}+q_{4}\right) \delta t \theta\left(v^{2} v_{x}\right)^{(n)}-\left(3 q_{3}+q_{4}\right) \delta t(1-\theta)\left(v^{2} v_{x}\right)^{(n)} \\
& +\delta t\left[\left(u_{x x}\right)^{(n)}+q_{1}\left(\left(u^{2}\right)^{(n)}+\left(v^{2}\right)^{(n)}\right) u^{(n)}+q_{2}\left(\left(u^{2}\right)^{(n)}\right.\right. \\
& \left.\left.+\left(v^{2}\right)^{(n)}\right)^{2} u^{(n)}-2 q_{3}\left(u v u_{x}\right)^{(n)}-q_{4}\left(u^{2} v_{x}\right)^{(n)}\right] .
\end{aligned}
$$

The RBFs approximations for the solutions $u$ and $v$ of Eqs. (2.6) are given by

$$
u^{(n)}\left(x_{i}\right)=\sum_{j=1}^{N} \lambda_{1 j}^{(n)} \psi\left(r_{i j}\right), \quad v^{(n)}\left(x_{i}\right)=\sum_{j=1}^{N} \lambda_{2 j}^{(n)} \psi\left(r_{i j}\right), \quad i=1,2, \ldots, N .
$$

Using Eqs. (2.16), the system of Eqs. (2.14)-(2.15), along with the boundary conditions (2.8) can be written in matrix form as

$$
\begin{aligned}
& \left(\mathbf{A}+\left(3 q_{3}+q_{4}\right) \delta t \theta\left(\left(\mathbf{u}^{2}\right)^{(\mathbf{n})} * \mathbf{D}_{\mathbf{1}}+\mathbf{2} \mathbf{u}^{(\mathbf{n})} \mathbf{u}_{\mathbf{x}}^{(\mathbf{n})} * \mathbf{A}\right)\right)_{\mathbf{1}}{ }^{(n+1)} \\
& =\left[\mathbf{A}+2\left(3 q_{3}+q_{4}\right) \delta t \theta\left(\mathbf{u}^{\mathbf{2}}\right)^{(\mathbf{n})} * \mathbf{D}_{\mathbf{1}}-\left(3 q_{3}+q_{4}\right) \delta t(1-\theta)\left(\mathbf{u}^{\mathbf{2}}\right)^{(\mathbf{n})} * \mathbf{D}_{\mathbf{1}}\right]^{\mathbf{1}_{\mathbf{1}}}{ }^{(n)} \\
& +\delta t\left[-\left(\mathbf{v}_{\mathbf{x x}}\right)^{(\mathbf{n})}-q_{1}\left(\left(\mathbf{u}^{2}\right)^{(\mathbf{n})}+\left(\mathbf{v}^{\mathbf{2}}\right)^{(\mathbf{n})}\right) \mathbf{v}^{(\mathbf{n})}-q_{2}\left(\left(\mathbf{u}^{2}\right)^{(\mathbf{n})}\right.\right. \\
& \left.\left.+\left(v^{2}\right)^{(n)}\right)^{2} \mathbf{v}^{(\mathbf{n})}-2 q_{3}\left(\mathbf{u v v}_{\mathbf{x}}\right)^{(\mathbf{n})}-q_{4}\left(\mathbf{v}^{2} \mathbf{u}_{\mathbf{x}}\right)^{(\mathbf{n})}\right]+\mathbf{f}^{(\mathbf{n}+\mathbf{1})}, \\
& \left(\mathbf{A}+\left(3 q_{3}+q_{4}\right) \delta t \theta\left(\left(\mathbf{v}^{\mathbf{2}}\right)^{(\mathbf{n})} * \mathbf{D}_{\mathbf{1}}+\mathbf{2} \mathbf{v}^{(\mathbf{n})} \mathbf{v}_{\mathbf{x}}^{(\mathbf{n})} * \mathbf{A}\right)\right)_{\mathbf{2}}^{(n+1)} \\
& =\left(\mathbf{A}+2\left(3 q_{3}+q_{4}\right) \delta t \theta\left(\mathbf{v}^{\mathbf{2}}\right)^{(\mathbf{n})} * \mathbf{D}_{\mathbf{1}}-\left(3 q_{3}+q_{4}\right) \delta t(1-\theta)\left(\mathbf{v}^{\mathbf{2}}\right)^{(\mathbf{n})} * \mathbf{D}_{\mathbf{1}}\right)_{\mathbf{2}}{ }^{(n)} \\
& +\delta t\left(\left(\mathbf{u}_{\mathbf{x x}}\right)^{(\mathbf{n})}+q_{1}\left(\left(\mathbf{u}^{2}\right)^{(\mathbf{n})}+\left(\mathbf{v}^{\mathbf{2}}\right)^{(\mathbf{n})}\right) \mathbf{u}^{(\mathbf{n})}+q_{2}\left(\left(\mathbf{u}^{2}\right)^{(\mathbf{n})}\right.\right. \\
& \left.\left.+\left(v^{2}\right)^{(n)}\right)^{2} \mathbf{u}^{(\mathbf{n})}-2 q_{3}\left(\mathbf{u v u}_{\mathbf{x}}\right)^{(\mathbf{n})}-q_{4}\left(\mathbf{u}^{2} \mathbf{v}_{\mathbf{x}}\right)^{(\mathbf{n})}\right)+\mathbf{g}^{(\mathbf{n}+\mathbf{1})},
\end{aligned}
$$

where '*' stands for component by component multiplication and

$$
\mathbf{A}=\left[\psi\left(r_{i j}\right)\right]_{i, j=1}^{N}, \mathbf{D}_{\mathbf{1}}=\left[\psi^{\prime}\left(r_{i j}\right)\right]_{i, j=1}^{N} .
$$

In more compact form we can write Eqs. (2.17)-(2.18) as

$$
\begin{aligned}
& \lambda_{1}^{(n+1)}=\mathbf{M}_{1}^{-1} \mathbf{N}_{1} \lambda_{1}^{(n)}+\mathbf{M}_{1}^{-1} \mathbf{F}^{(n+1)}, \\
& \lambda_{2}^{(n+1)}=\mathbf{M}_{2}^{-1} \mathbf{N}_{2} \lambda_{2}^{(n)}+\mathbf{M}_{2}^{-1} \mathbf{G}^{(n+1)},
\end{aligned}
$$

where $\lambda_{1}^{(0)}$ and $\lambda_{2}^{(0)}$ are the values at the initial time step.

$$
\begin{aligned}
\mathbf{M}_{\mathbf{1}} & =\left(\mathbf{A}+\left(3 q_{3}+q_{4}\right) \delta t \theta\left(\left(\mathbf{u}^{\mathbf{2}}\right)^{(\mathbf{n})} * \mathbf{D}_{\mathbf{1}}+\mathbf{2} \mathbf{u}^{(\mathbf{n})} \mathbf{u}_{\mathbf{x}}^{(\mathbf{n})} * \mathbf{A}\right)\right), \\
\mathbf{M}_{\mathbf{2}} & =\left(\mathbf{A}+\left(3 q_{3}+q_{4}\right) \delta t \theta\left(\left(\mathbf{v}^{2}\right)^{(\mathbf{n})} * \mathbf{D}_{\mathbf{1}}+\mathbf{2} \mathbf{v}^{(\mathbf{n})} \mathbf{v}_{\mathbf{x}}^{(\mathbf{n})} * \mathbf{A}\right)\right), \\
\mathbf{N}_{\mathbf{1}} & =\left(\mathbf{A}+2\left(3 q_{3}+q_{4}\right) \delta t \theta\left(\mathbf{u}^{2}\right)^{(\mathbf{n})} * \mathbf{D}_{\mathbf{1}}-\left(3 q_{3}+q_{4}\right) \delta t(1-\theta)\left(\mathbf{u}^{\mathbf{2}}\right)^{(\mathbf{n})} * \mathbf{D}_{\mathbf{1}}\right), \\
\mathbf{N}_{\mathbf{2}} & =\left(\mathbf{A}+2\left(3 q_{3}+q_{4}\right) \delta t \theta\left(\mathbf{v}^{\mathbf{2}}\right)^{(\mathbf{n})} * \mathbf{D}_{\mathbf{1}}-\left(3 q_{3}+q_{4}\right) \delta t(1-\theta)\left(\mathbf{v}^{\mathbf{2}}\right)^{(\mathbf{n})} * \mathbf{D}_{\mathbf{1}}\right), \\
\mathbf{f}^{(\mathbf{n}+\mathbf{1})} & =\left(f_{1}^{(n+1)}, 0,0, \ldots, f_{2}^{(n+1)}\right)^{T}, \quad \mathbf{g}^{(\mathbf{n}+\mathbf{1})}=\left(g_{1}^{(n+1)}, 0,0, \ldots, g_{2}^{(n+1)}\right)^{T},
\end{aligned}
$$




$$
\begin{aligned}
\mathbf{F}^{(\mathbf{n}+\mathbf{1})} & =\mathbf{f}^{(\mathbf{n}+\mathbf{1})}+\delta t\left(-\left(\mathbf{v}_{\mathbf{x x}}\right)^{(\mathbf{n})}-q_{1}\left(\left(\mathbf{u}^{2}\right)^{(\mathbf{n})}+\left(\mathbf{v}^{\mathbf{2}}\right)^{(\mathbf{n})}\right) \mathbf{v}^{(\mathbf{n})}\right. \\
& \left.-q_{2}\left(\left(\mathbf{u}^{2}\right)^{(\mathbf{n})}+\left(\mathbf{v}^{2}\right)^{(\mathbf{n})}\right)^{2} \mathbf{v}^{(\mathbf{n})}\right) \delta t\left[-2 q_{3}\left(\mathbf{u v v} \mathbf{v}_{\mathbf{x}}\right)^{(\mathbf{n})}-q_{4}\left(\mathbf{v}^{2} \mathbf{u}_{\mathbf{x}}\right)^{(\mathbf{n})}\right], \\
\mathbf{G}^{(\mathbf{n}+\mathbf{1})} & =\mathbf{g}^{(\mathbf{n}+\mathbf{1})}+\delta t\left(\left(\mathbf{u}_{\mathbf{x x}}\right)^{(\mathbf{n})}+q_{1}\left(\left(\mathbf{u}^{\mathbf{2}}\right)^{(\mathbf{n})}+\left(\mathbf{v}^{\mathbf{2}}\right)^{(\mathbf{n})}\right) \mathbf{u}^{(\mathbf{n})}\right. \\
& \left.+q_{2}\left(\left(\mathbf{u}^{2}\right)^{(\mathbf{n})}+\left(\mathbf{v}^{\mathbf{2}}\right)^{(\mathbf{n})}\right)^{2} \mathbf{u}^{(\mathbf{n})}\right)+\delta t\left[-2 q_{3}\left(\mathbf{u v u} \mathbf{u}_{\mathbf{x}}\right)^{(\mathbf{n})}-q_{4}\left(\mathbf{u}^{2} \mathbf{v}_{\mathbf{x}}\right)^{(\mathbf{n})}\right] .
\end{aligned}
$$

Eq. (2.16) can be written in matrix form as

$$
\mathbf{u}^{(n)}=\mathbf{A} \lambda_{1}^{(n)}, \quad \mathbf{v}^{(n)}=\mathbf{A} \lambda_{2}^{(n)} .
$$

Using Eq.(2.19) in Eq. (2.21), we get

$$
\begin{aligned}
& \mathbf{u}^{(n+1)}=\mathbf{A} \mathbf{M}_{\mathbf{1}}^{-\mathbf{1}} \mathbf{N}_{\mathbf{1}} \mathbf{A}^{-\mathbf{1}} \mathbf{u}^{(n)}+\mathbf{A} \mathbf{M}_{\mathbf{1}}^{-\mathbf{1}} \mathbf{F}^{(\mathbf{n}+\mathbf{1})}, \\
& \mathbf{v}^{(n+1)}=\mathbf{A} \mathbf{M}_{\mathbf{2}}^{-\mathbf{1}} \mathbf{N}_{\mathbf{2}} \mathbf{A}^{-\mathbf{1}} \mathbf{v}^{(n)}+\mathbf{A} \mathbf{M}_{\mathbf{2}}^{-\mathbf{1}} \mathbf{G}^{(\mathbf{n}+\mathbf{1})} .
\end{aligned}
$$

From here we can find the solution at any time level $n$.

\section{StABility OF THE SCHEME}

In this section, we discuss the stability analysis of the scheme (2.22), by using spectral norm of the amplification matrix. We assume that $\mathbf{u}, \mathbf{v}$ be the numerical and $\mathbf{u}^{*}, \mathbf{v}^{*}$ be the exact solutions of Eqs. (2.6). The error vectors $\varepsilon_{i}^{(n)}(i=1,2)$ are defined by $\varepsilon_{1}^{(n)}=\mathbf{u}^{(n)}-\mathbf{u}^{*(n)}, \varepsilon_{2}^{(n)}=\mathbf{v}^{(n)}-\mathbf{v}^{*(n)}$. Putting values from Eq. (2.22) in these equations, we arrive at the following equations

$$
\begin{aligned}
& \varepsilon_{1}^{(n+1)}=\mathbf{u}^{(\mathbf{n}+\mathbf{1})}-\mathbf{u}^{*(n+1)}=\mathbf{A} \mathbf{M}_{\mathbf{1}}^{-\mathbf{1}} \mathbf{N}_{\mathbf{1}} \mathbf{A}^{-\mathbf{1}} \varepsilon_{1}^{(n)}=\mathbf{E}_{\mathbf{1}} \varepsilon_{1}^{(n)}, \\
& \varepsilon_{2}^{(n+1)}=\mathbf{v}^{(\mathbf{n}+\mathbf{1})}-\mathbf{v}^{*(n+1)}=\mathbf{A} \mathbf{M}_{\mathbf{2}}^{-\mathbf{1}} \mathbf{N}_{\mathbf{2}} \mathbf{A}^{-\mathbf{1}} \varepsilon_{2}^{(n)}=\mathbf{E}_{\mathbf{2}} \varepsilon_{2}^{(n)},
\end{aligned}
$$

where $\mathbf{E}_{\mathbf{1}}=\mathbf{A} \mathbf{M}_{\mathbf{1}}^{-\mathbf{1}} \mathbf{N}_{\mathbf{1}} \mathbf{A}^{-\mathbf{1}}$ and $\mathbf{E}_{\mathbf{2}}=\mathbf{A} \mathbf{M}_{\mathbf{2}}^{-\mathbf{1}} \mathbf{N}_{\mathbf{2}} \mathbf{A}^{-\mathbf{1}}$ are the amplification matrices. For the scheme to remain stable, $\varepsilon_{i}^{(n)}$ must approach to zero, $(i=1,2)$ as $n \longrightarrow \infty$ i.e $\rho\left(\mathbf{E}_{\mathbf{1}}\right) \leq 1, \rho\left(\mathbf{E}_{\mathbf{2}}\right) \leq 1$, which is a condition of stability, where $\rho\left(\mathbf{E}_{\mathbf{1}}\right)$ and $\rho\left(\mathbf{E}_{\mathbf{2}}\right)$ represent spectral radii of the matrices $\mathbf{E}_{\mathbf{1}}$ and $\mathbf{E}_{\mathbf{2}}$ respectively. 
TABLE 1. Spectral radii $\rho\left(\mathbf{E}_{\mathbf{1}}\right)$ and $\rho\left(\mathbf{E}_{\mathbf{2}}\right)$ for various values of MQ shape parameter $c$, at time $t=0.2$, when time step size $\delta t=0.0001$, spatial step size $\delta x=0.4$, number of nodes $\mathrm{N}=201$, spatial interval [5,45], $q_{1}=\frac{1}{2}, q_{2}=\frac{-7}{4}, q_{3}=-1, q_{4}=-2$, corresponding to Problem 1 .

\begin{tabular}{ccc}
\hline $\mathrm{c}$ & $\rho\left(\mathbf{E}_{\mathbf{1}}\right)$ & $\rho\left(\mathbf{E}_{\mathbf{2}}\right)$ \\
0.10 & 1.00025 & 1.00038 \\
0.20 & 1.00035 & 1.00043 \\
0.30 & 1.00038 & 1.00032 \\
0.40 & 1.00000 & 1.00032 \\
0.50 & 1.00039 & 1.00034 \\
0.80 & 1.00040 & 1.00035 \\
0.90 & 1.00040 & 1.00036 \\
1.50 & 1.00038 & 1.00038 \\
2.00 & 1.00052 & 1.00164 \\
2.50 & 1.00646 & 1.08955 \\
3.00 & 3.34593 & 4.09048 \\
3.30 & 46874.5 & 16416.02 \\
\hline
\end{tabular}

TABLE 2. Conserved quantities $I_{1}, I_{2}$ and $I_{3}$ for various values of MQ shape parameter $c$, at time $t=0.2$, when time step size $\delta t=$ 0.0001 , spatial step size $\delta x=0.4$, number of nodes $\mathrm{N}=201$, spatial interval $[-5,45], q_{1}=\frac{1}{2}, q_{2}=\frac{-7}{4}, q_{3}=-1, q_{4}=-2$, corresponding to problem 1 .

\begin{tabular}{ccccc}
\hline $\mathrm{c}$ & $I_{1}$ & $I_{2}$ & $I_{3}$ & $L_{2}$ \\
0.10 & 2.189675 & 2.435363 & -5.569890 & $6.60587 \mathrm{E}-002$ \\
0.20 & 2.194867 & 2.570123 & -5.732372 & $9.24601 \mathrm{E}-003$ \\
0.30 & 2.196505 & 2.673238 & -5.823594 & $1.99296 \mathrm{E}-003$ \\
0.40 & 2.197113 & 2.722172 & -5.862737 & $4.17283 \mathrm{E}-004$ \\
0.50 & 2.197341 & 2.742960 & -5.878324 & $6.90097 \mathrm{E}-005$ \\
0.80 & 2.197476 & 2.756321 & -5.887649 & $9.76190 \mathrm{E}-005$ \\
0.90 & 2.197482 & 2.756920 & -5.888028 & $1.24626 \mathrm{E}-004$ \\
1.50 & 2.197488 & 2.757416 & -5.888330 & $1.72375 \mathrm{E}-004$ \\
2.00 & 2.197489 & 2.757430 & -5.888339 & $1.77317 \mathrm{E}-004$ \\
2.50 & 2.197489 & 2.757432 & -5.888341 & $1.87258 \mathrm{E}-004$ \\
3.00 & 2.197487 & 2.757434 & -5.888338 & $2.46310 \mathrm{E}-004$ \\
3.30 & 2.197487 & 2.757440 & -5.888344 & $7.55716 \mathrm{E}-004$ \\
3.40 & 2.197467 & 2.757423 & -5.888275 & $1.61953 \mathrm{E}-003$ \\
3.50 & 2.197461 & 2.757449 & -5.888238 & $3.22212 \mathrm{E}-003$ \\
3.60 & 2.197450 & 2.757574 & -5.888219 & $7.13120 \mathrm{E}-003$ \\
3.70 & 2.197212 & 2.759948 & -5.887675 & $1.73985 \mathrm{E}-002$ \\
3.80 & 2.197143 & 2.855818 & -5.886441 & $7.13152 \mathrm{E}-002$ \\
3.90 & 7.985062 & 9815.303 & -28.67049 & $2.02353 \mathrm{E}+001$ \\
\hline
\end{tabular}



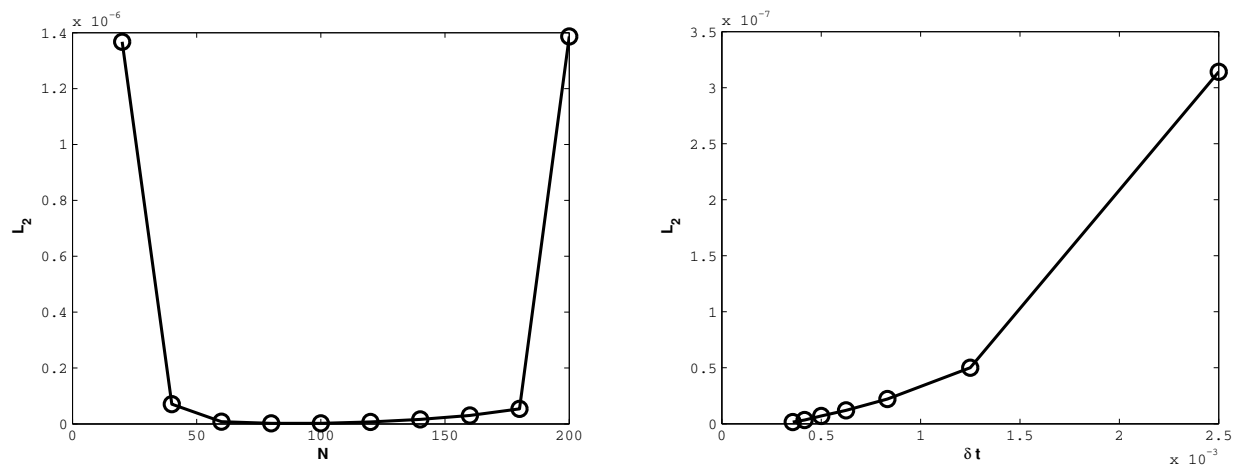

FIGURE 1. Plots, show $L_{2}$ error norms, versus $\mathrm{N}$ (Number of nodes), and temporal step size $\delta t$, at time $t=0.2$, time step size $\delta t=0.0001$, spatial step size $\delta x=0.4$, number of nodes $\mathrm{N}=201$, spatial interval $[-5,45], q_{1}=\frac{1}{2}, q_{2}=\frac{-7}{4}, q_{3}=-1, q_{4}=-2$, corresponding to Problem 1.

\section{NUMERICAL EXAMPLES}

In this section, we apply the proposed method for the numerical solution of GNS equation. The accuracy of the meshfree method is tested in terms of the three invariants of GNS equation given in Eq. (1.2) and the error norms $L_{2}, L_{\infty}$ defined as

$$
\begin{aligned}
& L_{2}=\left\|w^{*}-w\right\|_{2}=\left[\delta x \sum_{j=1}^{N}\left(w^{*}-w\right)^{2}\right]^{1 / 2}, \\
& L_{\infty}=\left\|w^{*}-w\right\|_{\infty}=\max \left|w^{*}-w\right| .
\end{aligned}
$$

The tested problems are given below.

\section{Problem 1: Single soliton}

We consider GNS equation (1.1) and choose the initial condition from the exact solution (1.5)

$$
\begin{aligned}
& w(x, 0)=\left[\frac{4}{4+3 \sinh ^{2}(x-15)}\right]^{1 / 2} \exp [i \phi(x, 0)] . \\
& \phi(x, 0)=2 \tanh ^{-1}\left[\frac{1}{2} \tanh (x-15)\right]+x-15
\end{aligned}
$$

In this problem the solution of GNS Eq. (1.1) represents a solitary wave initially centered at $x_{0}=15$. The exact values of the three invariants are $I_{1}=2 \log 3$, $I_{2}=-3 / 2+3.875 \log 3$ and $I_{3}=4-9 \log 3$, respectively. 
We solved the problem on the spatial interval, $-20 \leq x \leq 60$ and the time interval $[0,3]$, using three radial basis function, MQ, $r^{5}$ and TPS. The $L_{2}, L_{\infty}$ error norms and the three invariants of motion $I_{1}, I_{2}$ and $I_{3}$ are presented in Table 3 . We observe that better results are obtained, when MQ is used as compared to spline basis $r^{5}$ and TPS, and all the three invariants are very well preserved.

In Fig. 2(A), The relation between $L_{2}$ error norm and the number of collocation points $N$, for fixed value of the time step $\delta t=0.0001$, is presented. We observed that the solution converges for the choice of the nodes between, $N=40$ to $N=180$. In Fig. 2(B), $L_{2}$ error norm for various values of time step $\delta t$, and for fixed value of $N=201$ is shown.

In Fig. 3(A)-3(B), motion of the solitary wave is shown. The soliton, which is initially centered at $x_{0}=15$, moves from left to right, with constant speed and amplitude. Hence the motion of solitary wave is well resolved by the present method. For the purpose of comparison, we presented the three invariants, $I_{1}, I_{2}, I_{3}$ and $L_{2}$ error norm, in Table 4 . In this case, we chose constants $q_{1}=\frac{1}{2}, q_{2}=\frac{-7}{4}, q_{3}=-1, q_{4}=$ -2 , spatial interval $-5 \leq x \leq 45$, and time interval $[0,3]$. Table 4 , show that the present method preserved all the three invariants, when all the three radial basis functions are used, and are agreed with results obtained by orthogonal spline collocation method [23], however the present method conserved the second invariant $I_{2}$ more accurately than orthogonal spline collocation method [23]. In case of $L_{2}$ error norm, MQ radial basis function shown better accuracy than orthogonal spline collocation method [23]. 
TABLE 3. Error norms and Invariants for single soliton $|w|$, when time step size $\delta t=0.0001$, spatial step size $\delta x=0.4$, number of nodes $N=201$, spatial interval [-20,60], $q_{1}=\frac{1}{2}, q_{2}=\frac{-7}{4}, q_{3}=-1$, $q_{4}=-2$, and MQ shape parameters $c_{1}=c_{2}=2$, corresponding to the Problem 1.

\begin{tabular}{|c|c|c|c|c|c|c|}
\hline & MQ & & $r^{5}$ & & TPS & \\
\hline $\mathrm{t}$ & $L_{\infty}$ & $L_{2}$ & $L_{\infty}$ & $L_{2}$ & $L_{\infty}$ & $L_{2}$ \\
\hline 0.0001 & $1.55 \mathrm{e}-04$ & $5.58 \mathrm{e}-08$ & $1.55 \mathrm{E}-04$ & $6.03 \mathrm{E}-07$ & $1.55 \mathrm{E}-04$ & 2.93E-06 \\
\hline 0.2 & $2.45 \mathrm{e}-04$ & $1.80 \mathrm{e}-04$ & $7.60 \mathrm{E}-03$ & $1.59 \mathrm{E}-04$ & $2.10 \mathrm{E}-02$ & $4.08 \mathrm{E}-04$ \\
\hline 0.4 & $2.34 \mathrm{e}-04$ & $3.70 \mathrm{e}-04$ & $1.18 \mathrm{E}-02$ & $3.61 \mathrm{E}-04$ & $3.46 \mathrm{E}-02$ & $2.44 \mathrm{E}-03$ \\
\hline 0.6 & $4.24 \mathrm{e}-04$ & $8.09 \mathrm{e}-04$ & $1.37 \mathrm{E}-02$ & $2.17 \mathrm{E}-03$ & 4.15E-02 & $1.10 \mathrm{E}-02$ \\
\hline 0.8 & $3.50 \mathrm{E}-04$ & $1.74 \mathrm{E}-03$ & $1.54 \mathrm{E}-02$ & $8.02 \mathrm{E}-03$ & $4.84 \mathrm{E}-02$ & $3.04 \mathrm{E}-02$ \\
\hline \multirow[t]{3}{*}{1.0} & 4.62E-04 & $2.54 \mathrm{E}-03$ & $1.68 \mathrm{E}-02$ & $1.60 \mathrm{E}-02$ & $5.60 \mathrm{E}-02$ & $5.91 \mathrm{E}-02$ \\
\hline & MQ & & & $r^{5}$ & & \\
\hline & $I_{1}$ & $I_{2}$ & $I_{3}$ & $I_{1}$ & $I_{2}$ & $I_{3}$ \\
\hline 0.0001 & 2.1972 & 2.7571 & -5.8875 & 2.1972 & 2.7528 & -5.8852 \\
\hline 0.6 & 2.1980 & 2.7580 & -5.8900 & 2.1979 & 2.7562 & -5.8883 \\
\hline 1.0 & 2.1986 & 2.7587 & -5.8917 & 2.1984 & 2.7570 & -5.8900 \\
\hline 1.6 & 2.1994 & 2.7596 & -5.8942 & 2.1992 & 2.7580 & -5.8925 \\
\hline 2.0 & 2.1999 & 2.7602 & -5.8959 & 2.1997 & 2.7585 & -5.8942 \\
\hline 2.6 & 2.2007 & 2.7611 & -5.8984 & 2.2005 & 2.7593 & -5.8966 \\
\hline 3.0 & 2.2012 & 2.7617 & -5.9001 & 2.2010 & 2.7598 & -5.8982 \\
\hline
\end{tabular}

TABLE 4. Invariants and $L_{2}$ error norm for Single soliton, time step size $\delta t=0.0001$, spatial step size $\delta x=0.4$, number of nodes $N=$ 126, spatial interval $[-5,45], q_{1}=\frac{1}{2}, q_{2}=\frac{-7}{4}, q_{3}=-1, q_{4}=-2$, and MQ shape parameters $c_{1}=c_{2}=2$, corresponding to Problem 1 .

\begin{tabular}{ccccc}
\hline $\mathrm{t}$ & $I_{1}$ & $I_{2}$ & $I_{3}$ & $L_{2}$ \\
\hline $\mathrm{MQ}$ & & & & \\
\hline 0 & 2.197225 & 2.757123 & -5.887504 & $5.837 \mathrm{E}-011$ \\
1 & 2.198555 & 2.758655 & -5.891696 & $1.837 \mathrm{E}-003$ \\
2 & 2.199890 & 2.760191 & -5.895901 & $3.842 \mathrm{E}-003$ \\
3 & 2.201225 & 2.761726 & -5.900107 & $5.691 \mathrm{E}-003$ \\
\hline $\mathrm{TPS}$ & & & & \\
\hline 0 & 2.197225 & 2.742761 & -5.879475 & $1.797 \mathrm{E}-005$ \\
1 & 2.197724 & 2.761787 & -5.888847 & $5.841 \mathrm{E}-002$ \\
2 & 2.200530 & 2.797950 & -5.880088 & $3.963 \mathrm{E}-001$ \\
3 & 2.206579 & 2.854238 & -5.848961 & $6.603 \mathrm{E}-001$ \\
\hline$r^{5}$ & & & & \\
\hline 0 & 2.197225 & 2.752753 & -5.885190 & $3.032 \mathrm{E}-008$ \\
1 & 2.198397 & 2.757003 & -5.890038 & $1.557 \mathrm{E}-002$ \\
2 & 2.199696 & 2.758516 & -5.894150 & $6.436 \mathrm{E}-002$ \\
3 & 2.201017 & 2.759837 & -5.898085 & $9.878 \mathrm{E}-002$ \\
\hline OSC [23] & & & & \\
\hline 0 & 2.190546 & 2.861766 & -5.873885 & $1.451 \mathrm{E}-002$ \\
1 & 2.192070 & 2.917542 & -5.881301 & $1.837 \mathrm{E}-002$ \\
2 & 2.192500 & 2.970853 & -5.882713 & $2.320 \mathrm{E}-002$ \\
3 & 2.193853 & 3.242514 & -5.881409 & $3.390 \mathrm{E}-002$ \\
\hline
\end{tabular}



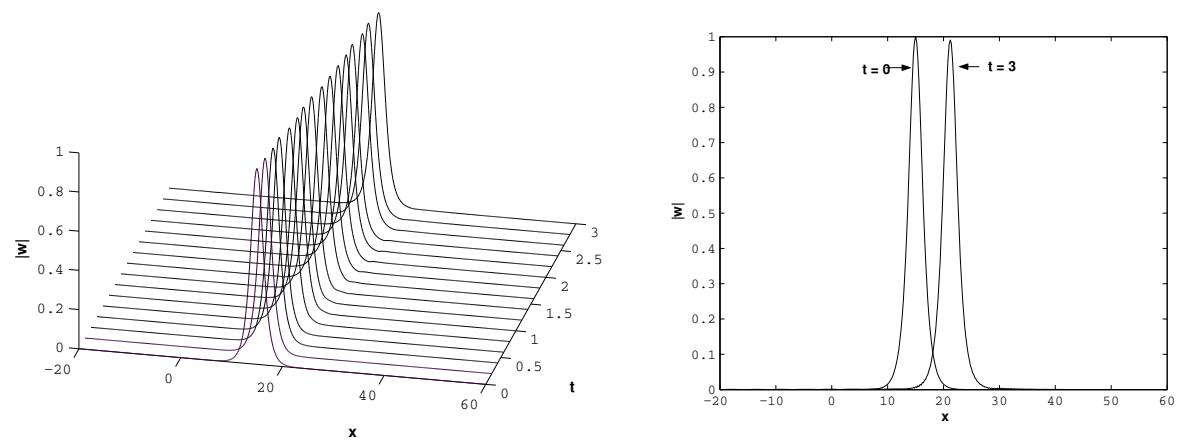

FIGURE 2. Motion of solitary wave $|w|$, for time step size $\delta t=$ 0.0001 , spatial step size $\delta x=0.4$, number of nodes $N=201$, spatial interval $[-20,60], q_{1}=\frac{1}{2}, q_{2}=\frac{-7}{4}, q_{3}=-1, q_{4}=-2$, and MQ shape parameters $c_{1}=c_{2}=2$, corresponding to problem 1 .

\section{Problem 2: Blow-up}

Here we present a test problem for the GNS equation. In [21] it has been shown that the exact solution of GNS will blow up in finite time for some specific values of the coefficients $q_{1}=-2, q_{2}=20, q_{3}=q_{4}=0$ and the Gaussian initial condition $w(x, 0)=e^{-x^{2}}$. For this problem the exact values of the three invariants are $I_{1}=$ $\sqrt{\pi / 2} \approx 1.253, I_{2}=\sqrt{\pi}(9 \sqrt{2}+9-20 \sqrt{6}) / 18 \approx-2.684$, and $I_{3}=0$. We applied two radial basis functions for the solution of the problem on the interval $[-7.5,7.5]$ for time up to $t=0.075$. The numerical values of the three invariants $I_{1}, I_{2}, I_{3}$ and the maximum modulus of the approximate solution $|w(x, 0)|$, for each time $\mathrm{t}$, are shown in Table 5 and Fig. 4. The same problem has also been investigated numerically by G.M. Muslu [19] using Higher-order split-step Fourier method, and M. P. Robinson [23] using an orthogonal spline collocation method. The results obtained by these methods are given in Table 5. From the comparison, we observed that the results obtained by these different methods, about the predicted time of blow up are fully consistent, which show accuracy and correctness of these methods. By the present method the blowup is quite evident near the time $\mathrm{t}=0.08$, and quite consistent with the results obtained in [23] and [19]. This blowup is accompanied by a radical change in the three invariants $I_{1}, I_{2}$ and $I_{3}$, and the maximum modulus of the approximate solution $|w(x, 0)|$, however $I_{2}$ changes more rapidly as compared to $I_{1}$ and $I_{3}$. 
TABLE 5. Variation of invariants and $|w(0, t)|$ in case of blow-up, time step size $\delta t=0.0001$, spatial step size $\delta x=0.1$, number of nodes $N=151$, spatial interval [-7.5,7.5], $q_{1}=-2, q_{2}=20, q_{3}=0$, $q_{4}=0$, and MQ shape parameters $c_{1}=c_{2}=0.1$, corresponding to Problem 2.

\begin{tabular}{ccccc}
\hline $\mathrm{t}$ & $I_{1}$ & $I_{2}$ & $I_{3}$ & $|w(0, t)|$ \\
\hline $\mathrm{MQ}$ & & & & \\
\hline 0.0 & 1.253314 & -2.684666 & 0.000000 & 1.000000 \\
0.01 & 1.253453 & -2.687043 & 0.000000 & 1.007541 \\
0.06 & 1.255209 & -3.210414 & 0.000000 & 1.527028 \\
0.07 & 1.267249 & -34.599645 & -0.000000 & 2.316339 \\
0.75 & 1.613581 & -500.855311 & 0.000000 & 3.115970 \\
\hline$r^{5}$ & & & & \\
\hline 0.0 & 1.253314 & -2.684467 & 0.000000 & 1.000000 \\
0.01 & 1.253454 & -2.685862 & 0.000000 & 1.007411 \\
0.06 & 1.255197 & -2.892629 & 0.000000 & 1.526226 \\
0.07 & 1.267809 & -26.934744 & 0.000008 & 2.325083 \\
0.075 & 1.582609 & -412.744272 & 0.000001 & 3.046048 \\
\hline OSC [23] & \multicolumn{5}{|c}{} & \\
\hline 0.00 & 1.253314 & -2.684467 & 0.000000 & 1.000000 \\
0.01 & 1.253154 & -2.682892 & $0.303(-13)$ & 1.007286 \\
0.06 & 1.253098 & -2.681302 & $-0.864(-09)$ & 1.525606 \\
0.07 & 1.252911 & -2.566558 & $0.220(-09)$ & 2.368234 \\
0.08 & 1.244698 & 34.08543 & $-0.675(-03)$ & 3.856267 \\
\hline SFS [19] & \multicolumn{5}{c}{} & \\
\hline 0.00 & 1.253314 & -2.684467 & $-9.993(-17)$ & 1.000000 \\
0.01 & 1.253314 & -2.684467 & $-6.651(-13)$ & 1.007348 \\
0.06 & 1.253314 & -2.684467 & $-4.673(-12)$ & 1.526243 \\
0.07 & 1.253314 & -2.684448 & $-4.395(-12)$ & 2.376429 \\
0.08 & 1.253335 & -2.829258 & $-6.085(-10)$ & 3.430374 \\
\hline & & & &
\end{tabular}



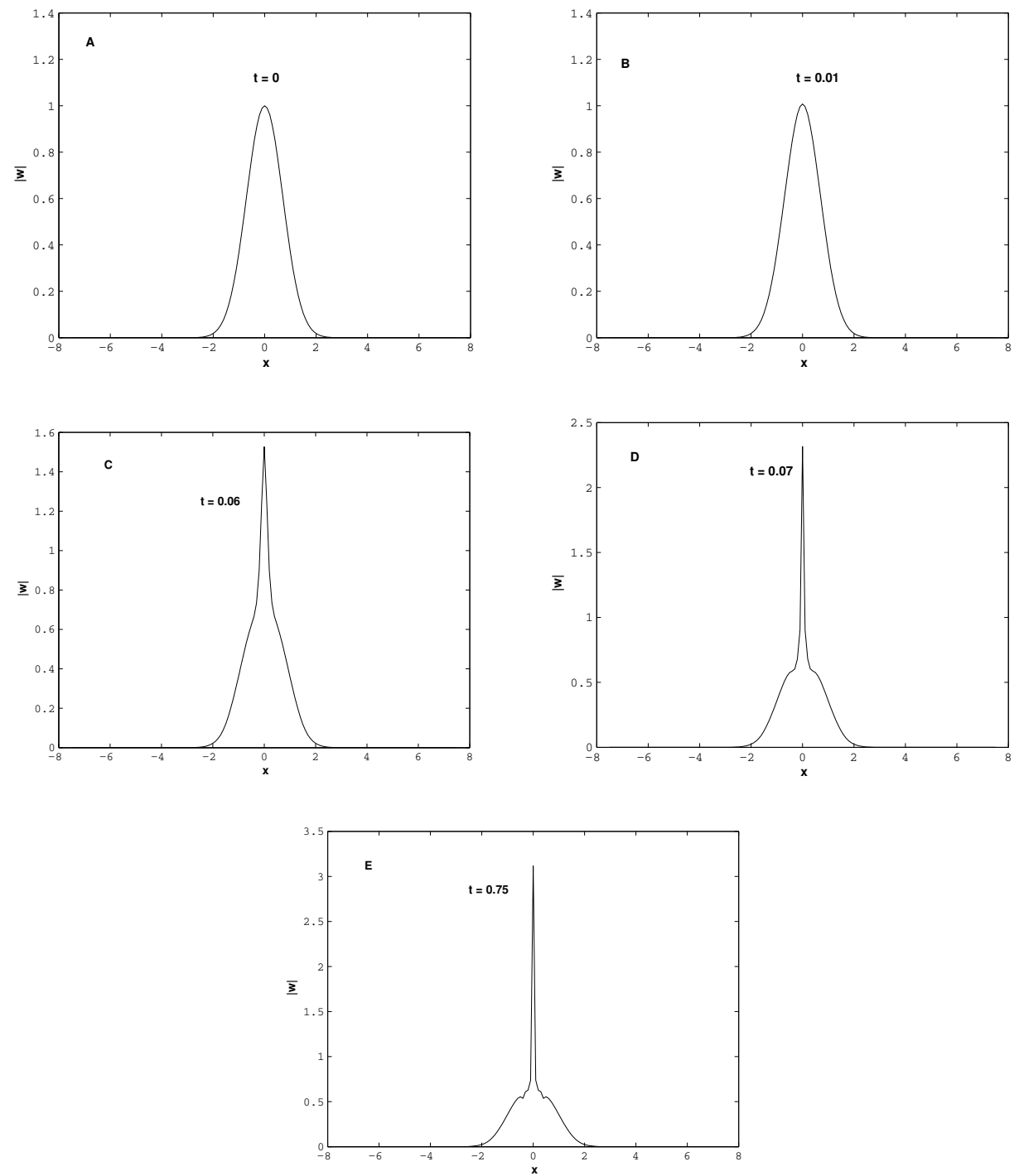

FiguRE 3. Graph of $|w(0, t)|$, at time $t=0.01,0.06,0.07,0.75$ in case of blow-up, time step size $\delta t=0.0001$, spatial step size $\delta x=$ 0.1 , number of nodes $N=151$, spatial interval [-7.5,7.5], $q_{1}=-2$, $q_{2}=20, q_{3}=0, q_{4}=0$, and MQ shape parameters $c_{1}=c_{2}=0.1$, corresponding to Problem 2. 


\section{Problem 3: Two soliton interaction}

Here we consider the interaction of two solitary waves, with the initial condition of the form

$$
\begin{aligned}
w(x, 0) & =1 / \sqrt{2} \operatorname{sech}\left[\frac{1}{2}\left(x-x_{1}\right)\right] \exp i\left[\frac{1}{4}\left(x-x_{1}\right)+\tanh \left(\frac{1}{2}\left(x-x_{1}\right)\right)\right] \\
& +1 / 2 \sqrt{2} \operatorname{sech}\left[-\frac{1}{4}\left(x-x_{2}\right)\right] \exp i\left[-\frac{1}{2}\left(x-x_{2}\right)+\frac{1}{2} \tanh \left(\frac{1}{4}\left(x-x_{2}\right)\right)\right] .
\end{aligned}
$$

This equation corresponds to sum of two solitary waves, the one initially located at $x_{1}=15$, while the other initially located at $x_{2}=35$ and moving in opposite directions. The problem is solved on the interval $-60 \leq x \leq 110$, for up to time $t=30$ by the meshfree method. For $q_{1}=1, q_{2}=1, q_{3}=-2$ and $q_{4}=0$, the interaction profile is shown in Fig. 6. We note that the two waves moves toward each other, collides with each other, and then moves away from each other as the time increases. We see that interaction, is consistent with [19]. The numerical values of the three invariants for this interaction of two waves up to time $t=30$ are presented in Table 5, by using two radial basis functions MQ and $r^{5}$. We can see that all the three invariants are preserved, and this behavior of the conserved quantity is a handy check on the accuracy of the present method.

\section{CONCLUDING REMARKS}

In this paper, a meshfree interpolation method using different types of RBFs is applied for the numerical solution of GNS equation. The method have shown excellent agreement with exact solution and the earlier work [19,23]. As a whole the present method produces better results with ease of implementation. The technique used in this paper provides an efficient alternative for the solution of nonlinear partial differential equations.

TABLE 6. Invariants for two soliton, $\delta t=0.0001, \delta x=1, N=$ $171,[-60,110], q_{1}=1, q_{2}=1, q_{3}=-2, q_{4}=0$, and MQ shape parameters $c_{1}=c_{2}=2$, corresponding to Problem 3 .

\begin{tabular}{ccccccc}
\hline \multicolumn{7}{c}{$\mathrm{MQ}$} \\
$\mathrm{t}$ & $I_{1}$ & $I_{2}$ & $I_{3}$ & $I_{1}$ & $I_{2}$ & $I_{3}$ \\
\hline 0.0001 & 3.00168 & 0.44296 & -0.75434 & 3.00168 & 0.44098 & -0.75159 \\
5 & 3.00180 & 0.44299 & -0.75439 & 2.99974 & 0.44584 & -0.75614 \\
10 & 3.00188 & 0.44303 & -0.75438 & 3.00073 & 0.44727 & -0.75390 \\
15 & 3.00197 & 0.44308 & -0.75432 & 2.99976 & 0.44765 & -0.75673 \\
20 & 3.00107 & 0.44314 & -0.75423 & 2.99979 & 0.44769 & -0.75648 \\
25 & 3.00214 & 0.44319 & -0.75427 & 2.99989 & 0.44768 & -0.75589 \\
30 & 3.00224 & 0.44326 & -0.75441 & 3.00008 & 0.44768 & -0.75501 \\
\hline
\end{tabular}




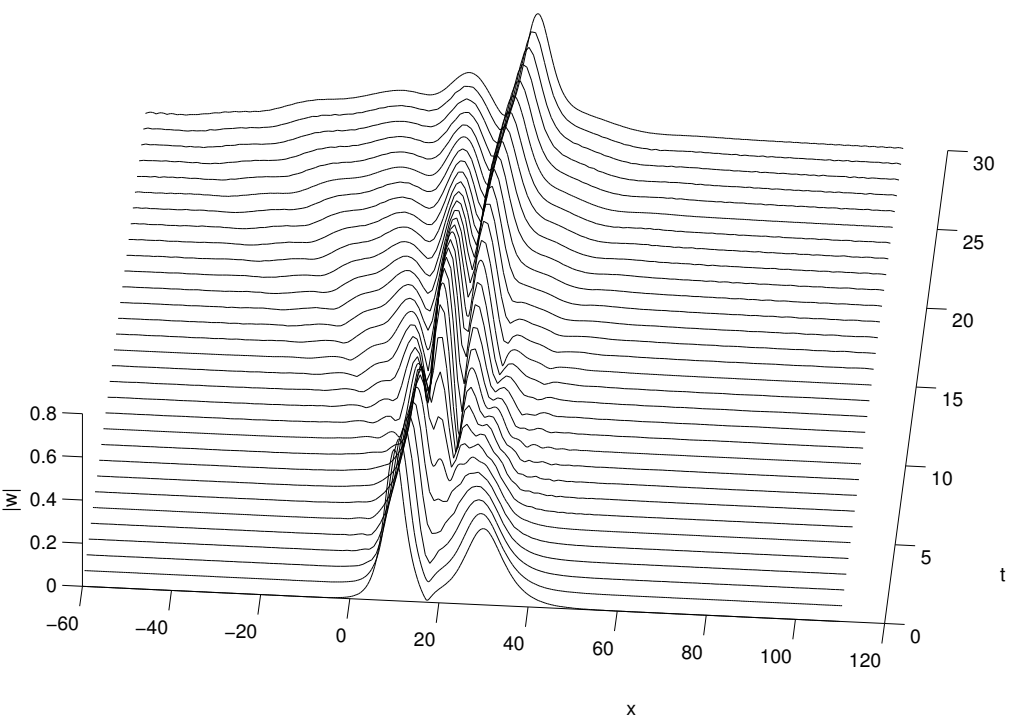

FIGURE 4. Interaction profile of two solitary waves, time step size $\delta t=0.001$, spatial step size $\delta x=1$, number of node $N=171$, spatial interval $[-60,110], q_{1}=1, q_{2}=1, q_{3}=-2, q_{4}=0$, and MQ shape parameters $c_{1}=c_{2}=2$, corresponding to problem 3 .

\section{ACKNOWLEDGEMENT}

The authors are thankful to the reviewers for their constructive comments.

\section{REFERENCES}

[1] G. Adomian and R. Meyers, "Generalized nonlinear Schrödinger equation with time-dependent dissipation," Appl. Math. Lett., vol. 8, no. 6, pp. 7-8, 1995.

[2] S. N. Atluri, Z. D. Han, and A. M. Rajendran, "A new implementation of the meshless finite volume method, through the MLPG "mixed" approach," CMES, Comput. Model. Eng. Sci., vol. 6, no. 6, pp. 491-513, 2004.

[3] Q. Chang, E. Jia, and W. Sun, "Difference schemes for solving the generalized nonlinear Schrödinger equation,” J. Comput. Phys., vol. 148, no. 2, pp. 397-415, art. no. jcph.1998.6120, 1999.

[4] A. Cloot, B. M. Herbst, and J. A. C. Weideman, "A numerical study of the nonlinear Schrödinger equation involving quintic terms," J. Comput. Phys., vol. 86, no. 1, pp. 127-146, 1990.

[5] A. Emdadi, E. J. Kansa, N. A. Libre, M. Rahimian, and M. Shekarchi, "Stable PDE solution methods for large multiquadric shape parameters," CMES, Comput. Model. Eng. Sci., vol. 25, no. 1, pp. 23-41, 2008.

[6] C. Franke and R. Schaback, "Convergence order estimates of meshless collocation methods using radial basis functions," Adv. Comput. Math., vol. 8, no. 4, pp. 381-399, 1998. 
[7] R. Franke, "A critical comparison of some methods for interpolation of scattered data," Naval Postgraduate School, Tech. Rep. NPS-53-79-003, 1975.

[8] R. Franke, "Scattered data interpolation: Tests of some methods," Math. Comput., vol. 38, pp. $181-200,1982$.

[9] M. A. Golberg, C. S. Chen, and S. R. Karur, "Improved multiquadric approximation for partial differential equations," Engng. Anal. Bound. Elemt., vol. 18, pp. 9-17, 1996.

[10] S. Haq, S. I. Islam, and A. Ali, "A numerical meshfree technique for the solution of mew equation," CMES: Comput. Model. Engin. Sci., vol. 38, pp. 1-23, 2008.

[11] R. L. Hardy, "Multiquadric equations of topography and other irregular surfaces," Geo. Phycs. Res., vol. 176, pp. 1905-1915, 1971.

[12] Q. He, "Domain decomposition method for nonlinear generalized Schrödinger-type systems: Semi-discrete problem," Appl. Math. Comput., vol. 77, no. 1, pp. 33-52, 1996.

[13] F. J. Hickernell and Y. C. Hon, "Radial basis function approximation of the surface wind field from scattered data," International Journal of applied science and Computations, vol. 4, pp. 221-247, 1998.

[14] E. J. Kansa, "Multiquadrics - a scattered data approximation scheme with applications to computational fluid-dynamics. I: Surface approximations and partial derivative estimates," Comput. Math. Appl., vol. 19, no. 8-9, pp. 127-145, 1990.

[15] G. Kosec and B. Sarler, "Local RBF collocation method for darcy flow," CMES: Comput. Model. Eng. Sci., vol. 25, pp. 197-207, 2008.

[16] N. A. Libre, A. Emdadi, E. J. Kansa, M. Shekarchi, and M. Rahimian, "A fast adaptive wavelet scheme in rbf collocation for nearly singular potential pdes," CMES: Comput. Model. Engin. Sci., vol. 38, pp. 263-284, 2008.

[17] W. R. Madych and S. A. Nelson, "Multivariate interpolation and conditionally positive definite functions. II," Math. Comput., vol. 54, no. 189, pp. 211-230, 1990.

[18] C. A. Micchelli, "Interpolation of scattered data: distance matrices and conditionally positive definite functions," Constr. Approx., vol. 2, pp. 11-22, 1986.

[19] G. M. Muslu and H. A. Erbay, "Higher-order split-step Fourier schemes for the generalized nonlinear Schrödinger equation,” Math. Comput. Simul., vol. 67, no. 6, pp. 581-595, 2005.

[20] P. Orsini, H. Power, and H. Morvan, "Improving volume element methods by meshless radial basis function techniques," CMES, Comput. Model. Eng. Sci., vol. 23, no. 3, pp. 187-208, 2008.

[21] D. Pathria, J. Li, and Morris, "Exact solutions for a generalized nonlinear Schrodinger equation," Phys. Scripta, vol. 39, pp. 673-679, 1989.

[22] D. Pathria, J. Li, and Morris, "Pseudo-spectral solution of nonlinear Schrodinger equation," $J$. Comput.Phys., vol. 87, pp. 108-125, 1990.

[23] M. P. Robinson, "The solution of nonlinear Schrödinger equations using orthogonal spline collocation," Comput. Math. Appl., vol. 33, no. 7, pp. 39-57, 1997.

[24] Q. Sheng, A. Q. M. Khaliq, and E. A. Al-Said, "Solving the generalized nonlinear Schrödinger equation via quartic spline approximation," J. Comput. Phys., vol. 166, no. 2, pp. 400-417, 2001.

[25] M. Uddin, S. Haq, and Siraj-ul-Islam, "A mesh-free numerical method for solution of the family of Kuramoto-Sivashinsky equations," Appl. Math. Comput., vol. 212, no. 2, pp. 458-469, 2009.

[26] M. Uddin, S. Haq, and Siraj-Ul-Islam, "Numerical solution of complex modified Korteweg-de Vries equation by mesh-free collocation method," Comput. Math. Appl., vol. 58, no. 3, pp. 566$578,2009$. 
Authors' addresses

Marjan Uddin

University of Engineering and Technology Peshawar, Department of Basics Sciences, 25000, Peshawar, Pakistan

E-mail address: marjankhan1@hotmail.com (corresponding author)

\section{Sirajul Haq}

GIK Institute of Technology Topi, Faculty of Engineering Sciences, Topi, Pakistan

E-mail address: siraj-jcs@yahoo.co.in 